\title{
Evolving role of PET/CT with different tracers in the evaluation of pulmonary neuroendocrine tumours
}

\author{
Giorgio Treglia $•$ Luca Giovanella $•$ Filippo Lococo
}

Received: 29 December 2013 / Accepted: 2 January 2014 / Published online: 21 February 2014

(C) Springer-Verlag Berlin Heidelberg 2014

Neuroendocrine tumours (NETs) of the lung arise from bronchial mucosal cells known as enterochromaffin cells or Kulchitsky cells, which are part of the diffuse neuroendocrine system. These tumours account for $20-25 \%$ of all primary lung neoplasms [1]. The pathological spectrum of pulmonary NETs ranges from low- or intermediate-grade neoplasms, also known as typical or atypical bronchial carcinoids (BCs), respectively, to more aggressive tumours such as large cell and small cell lung cancer $[1,2]$. In detail, the pathological distinction is substantially based on the microscopic morphology, the grade of differentiation, the mitotic rate and the presence of necrosis [2].

The distinct features among the different pulmonary NETs include not only their pathological characteristics but also the clinical behaviour, epidemiology, treatment and prognosis [3]. From a clinical point of view, typical BCs are indolent neoplasms with a good prognosis, whereas atypical BCs have a less indolent behaviour with a certain propensity for metastatic spread. Both these well-differentiated pulmonary NETs are optimally treated with complete surgical excision, although some divergent management strategies of care have been recently recommended for typical and atypical $\mathrm{BCs}[4,5]$. Indeed, while surgical resection remains the gold standard in the treatment of BCs, the extension of parenchymal resection (lobar versus sublobar resection) and the need for a radical lymph node dissection may be influenced by the histological characterization $[4,5]$. Conversely, more aggressive

\footnotetext{
G. Treglia $(\bowtie) \cdot$ L. Giovanella

Department of Nuclear Medicine and PET/CT Center, Oncology Institute of Southern Switzerland, via Ospedale, 12, 6500 Bellinzona, Switzerland

e-mail: giorgiomednuc@libero.it

F. Lococo

Unit of Thoracic Surgery, IRCCS-Arcispedale Santa Maria Nuova, Reggio Emilia, Italy
}

pulmonary NETs, such as large cell and small cell lung cancer, often present with local invasion, thoracic lymph node metastases and distant spread; as a result, affected patients may not be candidates for surgical resection, often being treated with chemotherapy with or without radiation therapy, showing a poor prognosis $[3,4]$.

Therefore, a correct pathological identification of pulmonary NETs during the preoperative setting is a key element for planning the best strategy of care, considering the different biological behaviour of the various pulmonary NET subtypes. Nevertheless, the preoperative pathology differentiation between typical and atypical BCs frequently appears not accurate. Furthermore, the structural imaging findings are often similar in typical and atypical BCs and, accordingly, a clear differentiation between these pulmonary NETs is not possible through radiological findings only [1-4].

Positron emission tomography (PET)/CT with different tracers, such as ${ }^{18} \mathrm{~F}$-fluorodeoxyglucose (FDG) and somatostatin analogues labelled with ${ }^{68} \mathrm{Ga}\left({ }^{68} \mathrm{Ga}\right.$-somatostatin analogues), may provide useful information for evaluating pulmonary NETs, including BCs [6].

The diagnostic performance of ${ }^{18} \mathrm{~F}-\mathrm{FDG}$ PET/CT has been reported to be superior in more aggressive pulmonary NETs (large cell and small cell lung cancer) compared to BCs, which are usually characterized by low growth $[7,8]$. False-positive findings of ${ }^{18} \mathrm{~F}$-FDG PET/CT due to inflammatory lesions are well known [6]. A recent study reported that atypical BCs usually show a higher ${ }^{18} \mathrm{~F}$-FDG uptake compared to typical $\mathrm{BCs}$, due to their more aggressive behaviour [9]. Moreover, ${ }^{18} \mathrm{~F}$-FDG PET/CT may provide useful prognostic information in patients with pulmonary NETs because high maximum standardized uptake values $\left(\mathrm{SUV}_{\max }\right)$ are associated with short survival and poor prognosis [7-9].

Since pulmonary NETs usually present an overexpression of somatostatin receptors on their cell surface, somatostatin receptor imaging has been widely used in the diagnosis, 
staging and restaging of these tumours [6]. Recently, PET/CT using somatostatin analogues (such as DOTANOC, DOTATOC, DOTATATE) labelled with ${ }^{68} \mathrm{Ga}$ has been increasingly used in patients with $\mathrm{BCs}$ due to its high diagnostic accuracy in NET detection [10]. Some false-negative results of somatostatin receptor PET/CT in pulmonary NETs may be due to tumours with low expression of somatostatin receptors. Conversely, inflammatory lesions may occasionally cause false-positive results due to the high expression of somatostatin receptors on the cell surface of the inflammatory cells [10]. Somatostatin receptor $\mathrm{PET} / \mathrm{CT}$ has been demonstrated to be superior compared to somatostatin receptor scintigraphy in evaluating lung NETs, due to the superior spatial resolution of PET imaging [6]. Furthermore, ${ }^{68} \mathrm{Ga}$-somatostatin analogues provided better results compared to the radiolabelled amino acid ${ }^{18} \mathrm{~F}$ dihydroxyphenylalanine (DOPA) in evaluating lung NETs [6], but literature reports specifically addressing the use of ${ }^{18}$ F-DOPA in pulmonary NETs are few [11].

Since different information may be obtained using various PET tracers, some authors have recently reported preliminary results on dual-tracer PET/CT imaging using ${ }^{68} \mathrm{Ga}$-somatostatin analogues and ${ }^{18}$ F-FDG in well-differentiated pulmonary NETs [12-15]. A retrospective study by Kayani et al. compared ${ }^{68} \mathrm{Ga}$-DOTATATE and ${ }^{18} \mathrm{~F}$-FDG PET/CT findings in 18 patients with pulmonary NETs, including $13 \mathrm{BCs}$. These authors demonstrated that typical BCs showed significantly higher uptake of ${ }^{68} \mathrm{Ga}$-somatostatin analogues and significantly lower uptake of ${ }^{18}$ F-FDG compared to pulmonary NETs of higher grade. No false-positive uptake of ${ }^{68} \mathrm{Ga}$-DOTATATE was reported in this study population, but there were three sites of false-positive uptake of ${ }^{18} \mathrm{~F}$-FDG due to inflammation [12]. Jindal et al. retrospectively evaluated the role of ${ }^{68} \mathrm{Ga}-$ DOTATOC and ${ }^{18} \mathrm{~F}$-FDG PET/CT in differentiating typical and atypical BCs. The overall detection rate of ${ }^{68} \mathrm{Ga}$ DOTATOC PET/CT for typical and atypical BCs was 100 and $86 \%$, respectively, with a median $\mathrm{SUV}_{\max }$ of 33 and 10.3 , respectively. The overall detection rate of ${ }^{18} \mathrm{~F}-\mathrm{FDG}$ $\mathrm{PET} / \mathrm{CT}$ for typical and atypical BCs was 54 and $100 \%$ with a median $\mathrm{SUV}_{\max }$ of 2.6 and 5.9, respectively [13].

In a study published in this issue of the European Journal of Nuclear Medicine and Molecular Imaging, Venkitaraman et al. performed the first prospective comparison of ${ }^{68} \mathrm{Ga}-$ DOTATOC and ${ }^{18} \mathrm{~F}$-FDG PET/CT in 32 patients with clinical suspicion of BCs. The overall sensitivity, specificity and accuracy of ${ }^{68} \mathrm{Ga}$-DOTATOC PET/CT in the diagnosis of BCs were $96.15,100$ and $96.87 \%$, respectively, whereas those of ${ }^{18}$ F-FDG PET/CT were 78.26, 11.1 and $59.37 \%$, respectively. The sensitivity of ${ }^{68} \mathrm{Ga}$-DOTATOC PET/CT was superior in patients with typical BCs compared to those with atypical BCs (100 versus $80 \%$, respectively), whereas the sensitivity of ${ }^{18} \mathrm{~F}$-FDG PET/CT was superior in patients with atypical BCs compared to those with typical BCs (100 versus $61.9 \%$ ) [15].
Overall, based on available literature data, ${ }^{68} \mathrm{Ga}$-somatostatin analogues seem to be the PET tracers of choice in the initial evaluation of patients with clinical suspicion of BC. In these patients, in order to detect a BC, PET/CT using ${ }^{68} \mathrm{Ga}$ somatostatin analogues could be performed primarily and, if negative, ${ }^{18} \mathrm{~F}$-FDG PET/CT could be performed subsequently. In fact, a negative PET/CT using ${ }^{68} \mathrm{Ga}$-somatostatin analogues may substantially rule out the presence of typical BCs, but it seems not sufficient to exclude the presence of atypical $\mathrm{BCs}$ (which occasionally may present low uptake of ${ }^{68} \mathrm{Ga}$-somatostatin analogues but are often ${ }^{18} \mathrm{~F}$-FDGavid tumours). The combination of PET/CT using ${ }^{68} \mathrm{Ga}$ somatostatin analogues and ${ }^{18} \mathrm{~F}$-FDG seems to be very useful in predicting the histology in patients with clinical suspicion of BCs, allowing a "tailored" therapeutic approach in such patients. Further large prospective studies and cost-effectiveness analyses are needed to validate this diagnostic strategy.

Conflicts of interest None.

\section{References}

1. de Guevara AC, Burigana F, Nicolini A, Lugnani F. Neuroendocrine tumors of the lung: histological classification, diagnosis, traditional and new therapeutic approaches. Curr Med Chem 2013. doi:10.2174/ 0929867321666131129125953.

2. Rindi G, Klersy C, Inzani F, Fellegara G, Ampollini L, Ardizzoni A, et al. Grading the neuroendocrine tumors of the lung: an evidencebased proposal. Endocr Relat Cancer 2013;21:1-16.

3. Benson RE, Rosado-de-Christenson ML, Martínez-Jiménez S, Kunin JR, Pettavel PP. Spectrum of pulmonary neuroendocrine proliferations and neoplasms. Radiographics 2013;33:1631-49.

4. Okoye CC, Jablons DM, Jahan TM, Kukreja J, Cardozo S, Yom SS. Divergent management strategies for typical versus atypical carcinoid tumors of the thoracic cavity. Am J Clin Oncol 2013. doi:10. 1097/COC.0b013e31827a7f6d.

5. Afoke J, Tan C, Hunt I, Zakkar M. Is sublobar resection equivalent to lobectomy for surgical management of peripheral carcinoid? Interact Cardiovasc Thorac Surg 2013;16:858-63.

6. Ambrosini V, Nicolini S, Caroli P, Nanni C, Massaro A, Marzola $\mathrm{MC}$, et al. PET/CT imaging in different types of lung cancer: an overview. Eur J Radiol 2012;81:988-1001.

7. Chong S, Lee KS, Kim BT, Choi JY, Yi CA, Chung MJ, et al. Integrated PET/CT of pulmonary neuroendocrine tumours: diagnostic and prognostic implications. AJR Am J Roentgenol 2007;188:1223-31.

8. Daniels CE, Lowe VJ, Aubry MC, Allen MS, Jett JR. The utility of fluorodeoxyglucose positron emission tomography in the evaluation of carcinoid tumors presenting as pulmonary nodules. Chest 2007;131:255-60.

9. Moore W, Freiberg E, Bishawi M, Halbreiner MS, Matthews R, Baram D, et al. FDG-PET imaging in patients with pulmonary carcinoid tumor. Clin Nucl Med 2013;38:501-5.

10. Treglia G, Castaldi P, Rindi G, Giordano A, Rufini V. Diagnostic performance of gallium- 68 somatostatin receptor PET and PET/CT in patients with thoracic and gastroenteropancreatic neuroendocrine tumours: a meta-analysis. Endocrine 2012;42:80-7.

11. Rufini V, Treglia G, Montravers F, Giordano A. Diagnostic accuracy of [18F]DOPA PET and PET/CT in patients with neuroendocrine tumors: a meta-analysis. Clin Transl Imaging 2013;1:111-22. 
12. Kayani I, Conry BG, Groves AM, Win T, Dickson J, Caplin M, et al. A comparison of 68Ga-DOTATATE and 18F-FDG PET/ CT in pulmonary neuroendocrine tumors. J Nucl Med 2009;50: 1927-32.

13. Jindal T, Kumar A, Venkitaraman B, Meena M, Kumar R, Malhotra A, et al. Evaluation of the role of [18F]FDG-PET/CT and [68Ga]DOTATOC-PET/CT in differentiating typical and atypical pulmonary carcinoids. Cancer Imaging 2011;11:70-5.
14. Paci M, Lococo F, Rapicetta C, Roncali M, Cavazza A, Treglia G, et al. Synchronous bilateral bronchial carcinoid diagnosed with combined dual tracer (18F-FDG and 68Ga-DOTATOC) PET/CT scans. Rev Esp Med Nucl Imagen Mol 2013. doi:10.1016/j.remn.2013.08.007.

15. Venkitaraman B, Karunanithi S, Kumar A, Khilnani GC, Kumar R. Role of $68 \mathrm{Ga}$-DOTATOC PET/CT in initial evaluation of patients with suspected bronchopulmonary carcinoid. Eur J Nucl Med Mol Imaging. doi:10.1007/s00259-013-2659-5. 